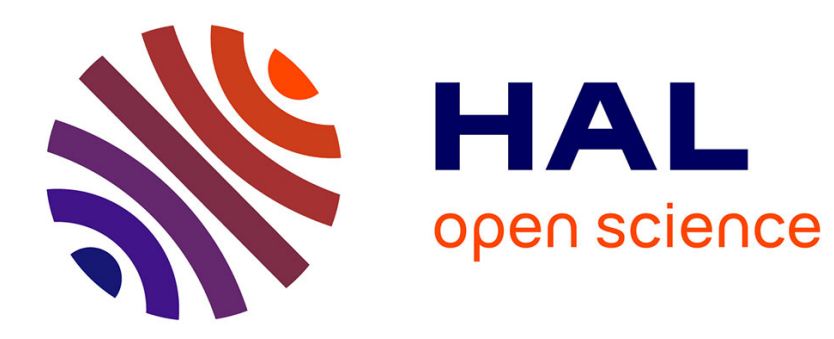

\title{
Relay Control Design for Robust Stabilization in a Finite-Time
}

Andrey Polyakov, Laurentiu Hetel

\section{To cite this version:}

Andrey Polyakov, Laurentiu Hetel. Relay Control Design for Robust Stabilization in a Finite-Time. IEEE Transactions on Automatic Control, 2016, 10.1109/TAC.2016.2591725 . hal-01347196

\author{
HAL Id: hal-01347196 \\ https://hal.inria.fr/hal-01347196
}

Submitted on 20 Jul 2016

HAL is a multi-disciplinary open access archive for the deposit and dissemination of scientific research documents, whether they are published or not. The documents may come from teaching and research institutions in France or abroad, or from public or private research centers.
L'archive ouverte pluridisciplinaire HAL, est destinée au dépôt et à la diffusion de documents scientifiques de niveau recherche, publiés ou non, émanant des établissements d'enseignement et de recherche français ou étrangers, des laboratoires publics ou privés. 


\title{
Relay Control Design for Robust Stabilization in a Finite-Time
}

\author{
Andrey Polyakov and Laurentiu Hetel
}

\begin{abstract}
The problem of robust finite-time stabilization of perturbed multi-input linear system by means of generalized relay feedback is considered. A new control design procedure, which combines convex embedding technique with Implicit Lyapunov Function (ILF) method, is developed. The sufficient conditions for both local and global finite-time stabilization are provided. The issues of practical implementation of the obtained implicit relay feedback are discussed. Our theoretical result is supported by numerical simulation for a Buck converter.
\end{abstract}

\section{INTRODUCTION}

Theory of relay automatic control systems has a long outstanding history. Relay feedbacks appeared in the early technological developments of the 19th century. However, the systematic theoretical study of relay control methods was provided in 1950s [1], [2]. The modern frequency domain approach to analysis and design of the relay systems can be found in [3].

When the sliding mode control methodology [4] was invented, it suggested to utilize a proper fast relay switching strategy in order to maintain the motion of the control system on a prescribed surface in the state space. Indeed, the classical example of the sliding mode system has the form of relay feedback: $\dot{x}(t)=-\operatorname{sign}[x(t)], t>0, x(0)=x_{0} \in \mathbb{R}$, where the sign function is defined as follows: $\operatorname{sign}[\rho]=1$ if $\rho>0$ and $\operatorname{sign}[\rho]=-1$ if $\rho<0$. Any trajectory of this system reaches the state $x=0$ in a finite time and remains thereafter. In fact, finite-time stability frequently accompanies the relay and sliding mode feedback systems [4], [5], [6], [7], [8]. The main application domains of sliding mode approach are electrical and electro-mechanical systems [4], [7]. Historically, being invented in 1960s the sliding mode is one of the first robust control approaches. Indeed, the sliding mode control is nonsensitive with respect to the so-called matched disturbances [9], [4].

Nowadays, the relay control systems can be found in different application domains. For instance, relay actuators (switches) are used in power converters [10] for control of output voltage but the relay (pulsed) jets are utilized in fluid dynamics for control of separated (turbulent) flows [11], [12].

The modern theoretical framework of hybrid dynamical systems [13], [14], [15] includes relay feedbacks as a particular case of switched affine systems [16], [17], [18], [19]. To the best of our knowledge, the finite-time stabilization problems have never been studied in the context of switched affine systems.

This research is supported by ANR ROCC-SYS (ANR-14-CE27-0008). A. Polyakov (andrey.polyakov@inria.fr) is with Non-A team-project, Inria Lille-Nord Europe, 40, avenue Halley, 59650 Villeneuve d'Ascq, France. L. Hetel (laurentiu.hetel@ec-lille.fr) and A. Polyakov are with CRIStAL UMR CNRS 9189, 59650 Villeneuve d'Ascq, France.
The present paper addresses the robust finite-time stabilization of linear multi-input system using the so-called generalized relay control. The generalized relay assumes a fixed set of possible values for the whole vector of control inputs [20]. This means, in particular, that the inputs cannot be switched independently. Such type of relay is motivated by practical demands of power electronics [21], where, for example, some switch cannot be turned on while an another one has the same state. Such restrictions in general case do not allow us to apply directly conventional schemes of relay sliding mode control design [4], [6]. In order to tackle this challenge, the technical note uses the convex embedding procedure. Recently, the ideas of convex embedding have been applied in order to design an locally exponentially stabilizing relay switching law based on the existence of a stabilizing linear feedback [20]. Here based on Implicit Lyapunov Function (ILF) method [22], [23], [24], [25] the convex embedding procedure allows us

- to design a finite-time stabilizing feedback for the case of generalized relays;

- to formulate stability conditions and restrictions to control parameters in terms of linear matrix inequalities;

- to obtain an estimate of both matched and mismatched uncertainties and disturbances, which can be rejected by the generalized relay feedback;

- to derive sufficient conditions for global finite-time stabilization and to estimate an attraction domain in the local case;

- to provide a settling-time estimate of the closed-loop system.

One more challenge tackled in the paper is the development of a feedback control rejecting state-dependent discontinuous disturbances. This allows us, for example, to take into account an unknown dry friction in mechanical or electromechanical models.

A preliminary version of this paper appeared in [26]. The key differences with respect to [26] are as follows: in this technical note we design a robust relay feedback control for perturbed multi-input system, present the proofs to all claims (Appendix), provide the global finite-time stabilization conditions (Proposition 7) and study a possible application of the proposed relay control to buck converter (Section VI).

The paper is organized as follows. The section II discusses problem statement and basic assumptions. After that preliminaries are considered. Next, the main result is presented. Finally, numerical simulation example and concluding remarks are given. All proofs are presented in Appendix.

Notation: $\mathbb{R}$ is the set of real numbers; $\mathbb{R}_{+}=\{x \in$ $\mathbb{R}: x>0\} ;\|x\|$ denotes the Euclidian norm of the vector 
$x \in \mathbb{R}^{n} ; \mathbb{R}$ ange $(B)$ is the column space of the matrix $B \in$ $\mathbb{R}^{n \times m} ; \operatorname{diag}\left\{\lambda_{1}, \ldots, \lambda_{n}\right\}$ is a diagonal matrix with elements $\lambda_{i}$; the order relation $P>0(<0, \geq 0, \leq 0)$ for $P \in \mathbb{R}^{n \times n}$ means that $P$ is symmetric and positive (negative) definite (semidefinite); if $P>0$ then the matrix $P^{1 / 2}:=B$ is such that $B^{2}=P ; \lambda_{\max }(P)$ and $\lambda_{\min }(P)$ denote maximum and minimum eigenvalues of the symmetric matrix $P \in \mathbb{R}^{n \times n}$; $\operatorname{co}(U)$ is the convex closure of the set $U \subset \mathbb{R}^{n} ; \operatorname{int}\{U\}$ denotes the interior of the set $U ; \mathcal{B}(r)=\left\{u \in \mathbb{R}^{m}:\|u\| \leq\right.$ $r\}$ is the ball of the radius $\varepsilon$ in $\mathbb{R}^{m}$.

\section{Problem Statement}

Let us consider a model of a control system described by the ordinary differential equation (ODE):

$$
\dot{x}(t)=A x(t)+B u(t)+d(t, x(t), u(t)), \quad t \in \mathbb{R}_{+},
$$

where $x(t) \in \mathbb{R}^{n}$ is the state vector, $u(t) \in \mathbb{R}^{m}$ is the vector of control inputs, $A \in \mathbb{R}^{n \times n}$ is the system matrix, $B \in \mathbb{R}^{n \times m}$ is the matrix of control gains and the locally measurable function $d: \mathbb{R}^{n+m+1} \rightarrow \mathbb{R}^{n}$ describes the exogenous disturbances and parametric uncertainties.

It is assumed that the matrices $A$ and $B$ are known, $\operatorname{rank}(B)=m \leq n$ and the pair $(A, B)$ is controllable; the whole state vector $x$ can be measured and utilized for control purposes. The control input $u$ is assumed to be a generalized relay, i.e. it can take values from a given discrete set:

$$
u(t) \in \mathcal{U}:=\left\{v_{1}, v_{2}, \ldots, v_{N}\right\}, \quad v_{i} \in \mathbb{R}^{m}, \quad t \in \mathbb{R}_{+},
$$

where $N$ is a natural number. In addition, the assumption

$$
0 \in \int\{\operatorname{co}(\mathcal{U})\} \subset \mathbb{R}^{m}
$$

is possessed in order to guarantee the existence of the locally stabilizing relay control (see, [20] for the details). As we will see further, this configuration includes as a particular case the classical sliding control generated by sign functions. This control configuration may also be related to the simplex method in [27], [28] and to the stabilization of switched affine systems [17], [18]. Filippov theory of differential equations with discontinuous right-hand sides [29] is utilized below in order to take into account the discontinuity of the control law and possible discontinuity of the disturbances.

The control aims are

- to stabilize the origin of the system (1) in a finite time,

- to describe a class of uncertainties $d$, which can be rejected by relay feedback control,

- to specify a set of admissible initial conditions (i.e. the domain of finite-time attraction).

Following the ideas of [20] the relay stabilizing control law can be designed in two steps. Initially, some (possibly continuous) stabilizing feedback should be selected. For this purpose the method of the Implicit Lyapunov Functions (ILF) is utilized [22], [24], [25], [30]. Next, a proper convex embedding procedure [20] is applied in order to construct the relay switching law in the form

$$
u(t) \in u_{r}(t, x(t))=\underset{v \in \mathcal{U}}{\operatorname{argmin}} \Gamma^{T}(t, x(t)) v,
$$

where $\Gamma: \mathbb{R}^{n+1} \rightarrow \mathbb{R}^{m}$ is a continuous (outside the origin) nonlinear function to be defined. The inclusion in (4) indicates that argmin is not unique in general case. In particular, if $m=1$ and $\mathcal{U}=\{-1,1\}$ then $u_{r}(t, x)=-\overline{\operatorname{sign}}[\Gamma(t, x)]$ similarly to the sliding mode control [4], where

$$
\overline{\operatorname{sign}}[\rho]=\left\{\begin{array}{ccc}
1 & \text { if } & \rho>0 \\
-1 & \text { if } & \rho<0 \\
\{-1,1\} & \text { if } & \rho=0 .
\end{array}\right.
$$

Note that in order to define the control input according to the formula (4) we just need to select the minimum of $\Gamma^{T}(t, x(t)) v$ over finite set values $v \in \mathcal{U}$. This operation does not need applying any finite or infinite dimensional optimization procedure. Just $N$ scalar products $\Gamma^{T}(t, x(t)) v_{i}, i=$ $1, \ldots, N$ must be calculated at each instant of time.

\section{PRELIMINARIES}

\section{A. Finite-Time Stability}

Let us consider the differential inclusion [29] of the form

$$
\dot{x}(t) \in F(t, x), \quad t \in \mathbb{R}_{+},
$$

where $x \in \mathbb{R}^{n}$ is the state vector, $F: \mathbb{R}^{n+1} \rightrightarrows \mathbb{R}^{n}$ is a multivalued map that is convex-valued, compact-valued and upper semi-continuous. It is well known [29] that an absolute continuous function $x:(a, b) \subset \mathbb{R}^{n}$ is called Caratheodory solution of (5) if it satisfies the differential inclusion (5) almost everywhere on the time interval $(a, b)$.

Let the origin be an equilibrium point of the system (5), i.e. $0 \in F(t, 0)$. Only strong uniform stability properties of the system (5) are studied in this paper, so the corresponding words "strong uniform" will be omitted below for shortness and simplicity of the presentation.

Definition 1 ([31], [32], [5]): The origin of system (5) is said to be finite-time stable if it is asymptotically stable and finite-time attractive, i.e. for any initial condition $x(0)=$ $x_{0} \in \mathcal{M} \backslash\{0\}$ there exists $T\left(x_{0}\right) \in \mathbb{R}_{+}$such that $x(t)=0$ for all $t \geq T\left(x_{0}\right)$, where $\mathcal{M}$ is a neighborhood of the origin and $T$ is called the settling-time function of the system (5). If $\mathcal{M}=\mathbb{R}^{n}$ then the origin is globally finite-time stable.

According to [29] the discontinuous system (1), (4) can also be treated as differential inclusion (5) properly constructed by means of the so-called Filippov regularization procedure. Below we deal only with Filippov solutions of the control system (1), (4).

\section{B. Implicit Lyapunov Function Method}

The next theorem is utilized below in order to design the feedback law.

Theorem 2: [25] If there exists a continuous function $Q$ : $\mathbb{R}_{+} \times \mathbb{R}^{n} \rightarrow \mathbb{R}$ that satisfies the conditions

C1) $Q$ is continuously differentiable in $\mathbb{R}_{+} \times \mathbb{R}^{n} \backslash\{0\}$;

$C 2)$ for any $x \in \mathbb{R}^{n} \backslash\{0\}$ there exist $V \in \mathbb{R}_{+}$such that $Q(V, x)=0$

C3) let $\Omega=\left\{(V, x) \in \mathbb{R}_{+} \times \mathbb{R}^{n}: Q(V, x)=0\right\}$ and

$$
\lim _{\substack{x \rightarrow 0 \\(V, x) \in \Omega}} V=0^{+}, \lim _{\substack{V \rightarrow 0^{+} \\(V, x) \in \Omega}}\|x\|=0, \lim _{\substack{\|x\| \rightarrow \infty \\(V, x) \in \Omega}} V=+\infty ;
$$


C4) the inequality $\frac{\partial Q(V, x)}{\partial V}<0$ holds for all $V \in \mathbb{R}_{+}$and $x \in \mathbb{R}^{n} \backslash\{0\}$;

C5) there exist $c \in \mathbb{R}_{+}$and $\mu \in(0,1]$ such that $\sup \frac{\partial Q(V, x)}{\partial x} y \leq c V^{1-\mu} \frac{\partial Q(V, x)}{\partial V},(V, x) \in \Omega ;$

$t \in \mathbb{R}_{+}, y \in F(t, x)$

then the origin of system $(5)$ is globally finite time stable with the following settling time estimate: $T\left(x_{0}\right) \leq \frac{V_{0}^{\mu}}{c \mu}$, where $V_{0} \in \mathbb{R}_{+}: Q\left(V_{0}, x_{0}\right)=0$.

Theorem 2 provides the sufficient conditions of finite-time stability for implicit definition of Lyapunov function. The conditions C1)-C4) guarantee existence and uniqueness of a continuously differentiable (outside the origin) positive definite radially unbounded function $V: \mathbb{R}^{n} \rightarrow \mathbb{R}_{+}$, which is implicitly defined by the equation $Q(V, x)=0$. The implicit function theorem [33] gives $\frac{\partial V}{\partial x}=-\left[\frac{\partial Q}{\partial V}\right]^{-1} \frac{\partial Q}{\partial x}$. Due to C4) and $C 5$ ) the estimate $\dot{V} \leq \sup _{t \in \mathbb{R}} \frac{\partial V}{\partial x} y \leq-c V^{1-\mu}$ implies the finite-time stability of the origin of (5) if $\mu \in(0,1]$. We refer the reader to [31], [23], [32], [5], [6], [25] for more details about finite-time stability and implicit Lyapunov function method.

Corollary 3: If the conditions C1)-C4) of Theorem 2 are fulfilled then the set

$$
\varepsilon\left(V_{0}\right)=\left\{z \in \mathbb{R}^{n}: Q\left(V_{0}, z\right) \leq 0\right\}
$$

is the $V_{0}$-level set $\left\{s \in \mathbb{R}^{n}: V(s) \leq V_{0}\right\}$ of the positive definite function $V: \mathbb{R}^{n} \rightarrow \mathbb{R}_{+}$implicitly defined by the equation $Q(V, s)=0$.

Proof: Indeed, if $\tilde{s} \in \mathbb{R}^{n}$ is such that $V(\tilde{s})=\alpha$, where $\alpha \in \mathbb{R}_{+}$then $Q(\alpha, \tilde{s})=0$, i.e. $\tilde{s} \in \varepsilon(\alpha)$. The condition C4) implies that $Q\left(V^{\prime}, \tilde{s}\right)<0$ (i.e $\tilde{s} \in \varepsilon\left(V^{\prime}\right)$ ) for any $V^{\prime}>\alpha$ and $Q\left(V^{\prime \prime}, \tilde{s}\right)>0$ (i.e. $\tilde{s} \notin \varepsilon\left(V^{\prime}\right)$ ) for any $V^{\prime \prime}<\alpha$.

Corollary 3 allows us to adapt Theorem 2 to local finitetime stability analysis possessing the condition C5) locally, i.e. $0<V<\bar{V}$ and $x \in \varepsilon(\bar{V})$ for some given $\bar{V} \in \mathbb{R}_{+}$. The level set $\varepsilon(\bar{V})$ specifies the finite-time attraction domain $\mathcal{M}$ in this case (see, Definition 1).

\section{IMPLICIT RELAY FEEDBACK LAW}

\section{A. Block Decomposition}

Let us initially decompose the original multi-input system (1) to a block form [34]. In order to make the paper selfcontained the block decomposition procedure studied in [35], [30] is very briefly discussed in Appendix. It constructs the non-singular coordinate transformation

$$
s=\Theta x
$$

reducing the original system (1) to the block form

$$
\dot{s}(t)=\tilde{A} s(t)+\tilde{B} u(t)+\tilde{d}(t, s(t), u(t)),
$$

where $\tilde{d}(t, s, u)=\Theta d\left(t, \Theta^{-1} s, u\right)+K_{l i n} s$ and $K_{l i n} \in \mathbb{R}^{n \times n}$,

$$
\tilde{A}=\left(\begin{array}{cccc}
0 & A_{12} & \ldots & 0 \\
\ldots & \ldots & \ldots & \ldots \\
0 & \ldots & \ldots & A_{k-1 k} \\
0 & \ldots & \ldots & 0
\end{array}\right), \quad \tilde{B}=\left(\begin{array}{c}
0 \\
\ldots \\
0 \\
A_{k k+1}
\end{array}\right) \in \mathbb{R}^{n \times n_{k}},
$$

the matrices $A_{i+1} \in \mathbb{R}^{n_{i} \times n_{i+1}}$ are of full row rank, $j=$ $1,2, . ., k, n_{1}+\ldots+n_{k}=n, n_{k}=m$ and $A_{k k+1} \in \mathbb{R}^{m \times m}$ is a nonsingular matrix, where $k$ is the number of blocks.

The function $\tilde{d}$ may be discontinuous. Following Filippov regularization procedure [29] we construct the compact and convex-valued upper semi-continuous map $\Delta: \mathbb{R}^{n+1} \rightrightarrows \mathbb{R}^{n}$ :

$$
\left.\Delta(t, s)=\bigcap_{\delta>0} \bigcap_{\mu(N)=0} c o(\tilde{d}(t, s+\mathcal{B}(\delta) \backslash N, c o(\mathcal{U})))\right),
$$

which collects all possible perturbations of the system (1).

\section{B. Relay Feedback Design}

Let us introduce the ILF function [30] of the form

$$
Q(V, s)=s^{T} D_{r}\left(V^{-1}\right) P D_{r}\left(V^{-1}\right) s-1,
$$

where $s=\left(s_{1}, \ldots, s_{k}\right)^{T}, s_{i} \in \mathbb{R}^{n_{i}}, V \in \mathbb{R}_{+}, D_{r}(\lambda)$ is the so-called homogeneous dilation matrix [30]

$$
D_{r}(\lambda)=\left(\begin{array}{cccc}
\lambda^{r_{1}} I_{n_{1}} & 0 & \ldots & 0 \\
0 & \lambda^{r_{2}} I_{n_{2}} & \cdots & 0 \\
\ldots & \ldots & \cdots & \ldots \\
0 & \cdots & 0 & \lambda^{r_{k}} I_{n_{k}}
\end{array}\right),
$$

where $\lambda \in \mathbb{R}_{+}$is a non-negative scaling parameter, $r_{i}=1+$ $(k-i) \mu, i=1,2, . ., k$ are scaling weights with $0<\mu \leq 1$, and $P \in \mathbb{R}^{n \times n}, P>0$. Denote $H_{\mu}:=\operatorname{diag}\left\{r_{i} I_{n_{i}}\right\}_{i=1}^{k}$.

Theorem 4: Let $\mu \in(0,1), \alpha>0, X \in \mathbb{R}^{n \times n}$ and $Y \in$ $\mathbb{R}^{n_{k} \times n}$ satisfy the system of matrix inequalities:

$$
\begin{gathered}
\tilde{A} X+X \tilde{A}^{T}+\tilde{B} Y+Y^{T} \tilde{B}^{T}+\alpha\left(H_{\mu} X+X H_{\mu}\right)+R \leq 0, \\
X H_{\mu}+H_{\mu} X>0, \quad X>0,
\end{gathered}
$$

then there exists a function $V: \mathbb{R}^{n} \rightarrow \mathbb{R}_{+}$implicitly defined by the equation $Q(V, s)=0$ with $Q$ is given by (11) for $P=X^{-1}$. Let $\beta \in(0, \alpha), V_{\max } \in \mathbb{R}_{+} \cup\{+\infty\}$ and the inequality

$$
\begin{gathered}
\delta^{T} D_{r}\left(V^{-1}\right) R^{-1} D_{r}\left(V^{-1}\right) \delta \leq \\
\beta V^{-2 \mu} s^{T} D_{r}\left(V^{-1}\right)\left(H_{\mu} P+P H_{\mu}\right) D_{r}\left(V^{-1}\right) s,
\end{gathered}
$$

holds for $\delta \in \Delta(t, s), V \in\left(0, V_{\max }\right), s \in \mathbb{R}^{n}: Q(V, s)=0$ and for almost all $t \in \mathbb{R}_{+}$, then the control (4) with

$$
\Gamma^{T}(t, x)=x^{T} \Theta^{T} D_{r}\left(\frac{1}{V(\Theta x)}\right) P D_{r}\left(\frac{1}{V(\Theta x)}\right) \tilde{B},
$$

locally stabilizes the origin of the system (1) in a finite time and the settling-time function $T$ can be estimated as follows

$$
T\left(x_{0}\right) \leq \frac{V_{0}^{\mu}}{(\alpha-\beta) \mu}, \quad \forall x_{0} \in \mathbb{R}^{n}: \Theta x_{0} \in \varepsilon(\bar{V}),
$$

where $V_{0} \in \mathbb{R}_{+}: Q\left(V_{0}, \Theta x_{0}\right)=0$ and $\varepsilon(\bar{V})$ is the finite-time attraction domain (6) with a positive $\bar{V} \in \mathbb{R}_{+}: \bar{V} \leq V_{\max }$.

All proofs are given in Appendix, where the estimate of the finite-time attraction domain is also provided.

The system of matrix inequalities (13) can be easily solved using LMI toolbox of MATLAB or, for example, SeDuMi solver. The solution of (13) also can be constructed analytically using the proof (see, [30]) of the next proposition.

Proposition 5 ([30]): If the pair $(A, B)$ is controllable then the system of matrix inequalities (13) is feasible for any $\mu \in \mathbb{R}_{+}$. 
Obviously, if $\tilde{d} \equiv 0$ then $\Delta \equiv\{0\}$ and (14) holds for any $R$. The matrix $R$ is introduced in (13) is order to characterize disturbance functions $d$, which do not affect the finite-time stability property of the closed-loop system. The formula (14) gives the implicit restrictions to the system disturbances. In order to provide the explicit ones let us introduce the matrices $E_{i}, i=1,2, . . k$ by the following formula

$$
E_{i}=\left(\begin{array}{ccccc}
0_{n_{1}} & \ldots & 0_{n_{1} \times n_{i}} & \ldots & 0_{n_{1} \times n_{k}} \\
\ldots & \ldots & \ldots & \ldots & \ldots \\
0_{n_{i} \times n_{1}} & \ldots & I_{n_{i}} & \ldots & 0_{n_{i} \times n_{k}} \\
\ldots & \ldots & \ldots & \ldots & \ldots \\
0_{n_{k} \times n_{1}} & \ldots & 0_{n_{k} \times n_{i}} & \ldots & 0_{n_{k}}
\end{array}\right) .
$$

Note that if $E_{i} \tilde{d} \neq 0$ just for some $i=i_{1}, i_{2}, \ldots, i_{p}$ then Theorem 4 stays true even when the term $R$ in the LMI (13) is replaced with $\left(E_{i_{1}}+E_{i_{2}}+\ldots+E_{i_{p}}\right) R\left(E_{i_{1}}+E_{i_{2}}+\ldots+E_{i_{p}}\right)$.

Proposition 6: Let $X \in \mathbb{R}^{n \times n}$ be a solution of the LMI system (13) with $R=\operatorname{diag}\left\{\beta_{1} I_{n_{1}}, \beta_{2} I_{n_{2}} \ldots, \beta_{k} I_{n_{k}}\right\}, \beta_{i} \in$ $\mathbb{R}_{+}: \beta=\beta_{1}+\ldots+\beta_{k}<\alpha$ and $P=X^{-1}$. If

$$
\delta^{T} E_{i} \delta \leq \beta_{i}^{2} \gamma\left\{\begin{array}{l}
\left(\lambda_{\min }(P) s^{T} s\right)^{1+(k-i-1) \mu} \text { if } s^{T} P s \leq 1, \\
\left(\lambda_{\min }(P) s^{T} s\right)^{\frac{1+(k-i-1) \mu}{1+(k-1) \mu}} \text { if } s^{T} P s>1,
\end{array}\right.
$$

for $\delta \in \Delta(t, s), \gamma=\lambda_{\min }\left(P^{-\frac{1}{2}}\left(P H_{\mu}+H_{\mu} P\right) P^{-\frac{1}{2}}\right)$ and $i=1,2, \ldots, k$ then the inequality (14) holds for any $V \in$ $\mathbb{R}_{+}: Q(V, s)=0$.

In the view of Corollary 3 the inequality $V \leq 1$ is equivalent to $s^{T} P s \leq 1$, so the restriction (18) can be used locally for $V_{\max }=1$ (see, (14)).

The class of the disturbances which can be rejected by the proposed control law is essentially depended on the parameter $\mu$. If $\mu=1$ then, obviously, condition (18) implies $\delta^{T} E_{k} \delta \leq \beta_{k}^{2} \gamma=$ const for any $s \in \mathbb{R}^{n}$, i.e. the constructed relay feedback rejects the so-called bounded matched disturbances [4]. The mismatched disturbances $E_{i} \delta, i=1,2, \ldots, k-1$ should vanish as $s \rightarrow 0$ and admit polynomial estimates close to the origin (see (18)).

Proposition 7: Theorem 4 stays true for $\mu=1$ if the matrix inequality (13) is supplied with the additional condition

$$
\left(\begin{array}{cc}
X & Y^{T} \\
Y & u_{0}^{2} I_{m}
\end{array}\right) \geq 0
$$

where $0<u_{0} \leq u_{\max }=\sup _{r \in \mathbb{R}_{+}: \mathcal{B}(r) \subset \operatorname{co}\{\mathcal{U}\}} r$ r. Moreover, the closed-loop system (1), (4), (15) is globally finite-time stable if $V_{\max }=+\infty$.

This proposition provides sufficient condition for global robust finite-time stability of the closed-loop relay system (1), (4), (15). In particular, it is globally finite-time stable if (13) and (19) holds and $\tilde{d}$ satisfies (18) for $\mu=1$.

\section{PRACTICAL IMPLEMENTATION}

In order to realize the control algorithm (4), (15) in practice we need to know $V$. In some cases the function $V$ can be calculated analytically [36]. The function $V$ can also be approximated numerically on a grid constructed in the finite-time attraction domain $\varepsilon(\bar{V})$. Finally, the relay control

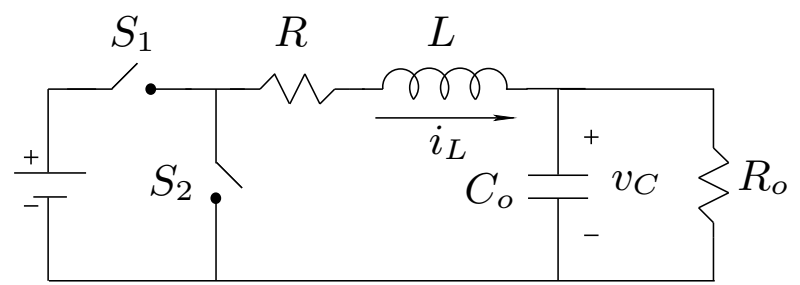

Fig. 1. Scheme of Buck converter

law (4) can be applied by means of on-line estimation of $V$. The following corollary may be utilized for this purpose.

Corollary 8: If 1) the conditions of Theorem 4 hold; 2) $\left\{t_{i}\right\}_{i=0}^{+\infty}$ is an arbitrary sequence of time instances such that $0=t_{0}<t_{1}<t_{2}<\ldots$ and $\left.\lim _{i \rightarrow+\infty} t_{i}=+\infty ; 3\right)$ the relay control $u_{r}$ has the form (4) with the sampled computation of the switching function $\Gamma(t, x)=\tilde{\Gamma}_{i}(\Theta x)$ for $t \in\left[t_{i}, t_{i+1}\right)$,

$$
\tilde{\Gamma}_{i}^{T}(s)=s^{T} D_{r}\left(V_{i}^{-1}\right) P D_{r}\left(V_{i}^{-1}\right) \tilde{B}, \quad Q\left(V_{i}, s\left(t_{i}\right)\right)=0 .
$$

Then the system (1), (4) is globally asymptotically stable.

The corollary shows that asymptotic stability of the closed loop system is maintained even if the switching function $\Gamma$ can be updated only at some isolated instants of time. The estimation of the switching parameter $V_{i}$ can be obtained using the simple bisection method. We refer the reader to [25], [30] for more details about practical implementation of the control algorithms based on implicit Lyapunov function.

\section{EXAMPle: CONTROL OF BUCK CONVERTER}

Let us consider the model of Buck converter (Fig. 1) studied in [10]: $x=\left(v_{C} i_{L}\right)^{T}, x \in \mathbb{R}^{2}, \mathcal{U}=\left\{0, v_{i n}\right\}$,

$$
A=\left(\begin{array}{cc}
-1 /\left(R_{o} C_{0}\right) & 1 / C_{o} \\
-1 / L & -R_{c} / L
\end{array}\right), B=\left(\begin{array}{c}
0 \\
1 / L
\end{array}\right),
$$

where $i_{L}$ - inductor current, $v_{C}$ - capacitor voltage, $v_{i n}=$ $100 \mathrm{~V}, R=2 \Omega, L=500 \mu \mathrm{H}, C_{o}=470 \mu \mathrm{F}$ and $R_{o}=$ $50 \Omega$. In the active mode, the switches $S_{1}$ and $S_{2}$ operate as follows: if $S_{1}$ is opened then $S_{2}$ is closed and vise versa.

Let us apply the coordinate transformation:

$s=\Theta\left(x-x^{*}\right), \quad \Theta=\left(\begin{array}{cc}1 & 0 \\ -1 / R_{o} & 1\end{array}\right), \quad x^{*}=\left(\begin{array}{c}v_{\text {out }} \\ v_{\text {out }} / R_{o}\end{array}\right)$,

where $v_{\text {out }} \in\left(0, v_{\text {in }} /\left(1+R_{c} / R_{o}\right)\right)$ is the required output voltage. We derive the following relay control system

$$
\dot{s}(t)=\left(\begin{array}{cc}
0 & 1 / C_{o} \\
0 & 0
\end{array}\right) s+B \hat{u}+\tilde{d}(s),
$$

where $\hat{u} \in\left\{v_{1}, v_{2}\right\}$,

$$
\begin{aligned}
& v_{1}=-v_{\text {out }}\left(1+R_{c} / R_{o}\right), \\
& v_{2}=v_{\text {in }}-v_{\text {out }}\left(1+R_{c} / R_{o}\right)
\end{aligned}
$$

and $\tilde{d}(s)=-\left(1 / L \quad R_{c} / L-1 /\left(R_{o} C_{o}\right)\right) s$. The obtained system satisfies the assumption (3) and all conditions of Theorem 4. The system of matrix inequalities (13), (19) gives

$$
P=\left(\begin{array}{ll}
0.1768 & 0.1172 \\
0.1172 & 0.1059
\end{array}\right), K=\left(\begin{array}{ll}
-0.0960 & -0.0707
\end{array}\right)
$$




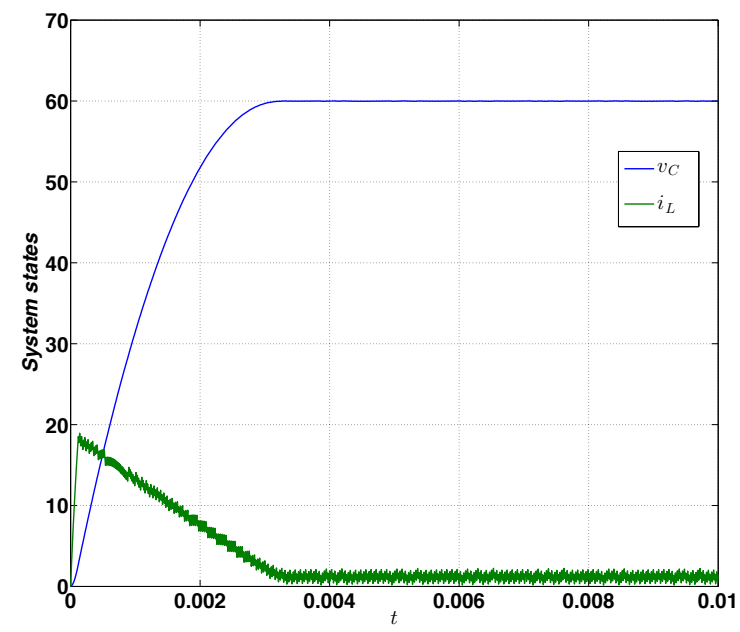

Fig. 2. Evolution of the system state

for the matrix $R=10^{5} I_{2} \in \mathbb{R}^{2 \times 2}$, for parameters $\mu=\alpha=1$ and $u_{0}=\min \left(\left|v_{1}\right|, v_{2}\right), v_{\text {out }}=60$. The switching surface $\Gamma$ is defined by (15) and the dilation matrix $D_{r}$ has the weights $r_{1}=2$ and $r_{2}=1$.

The Fig. 2 shows the simulation results of the control application for $x(0)=(0,0)^{T}$ and the sampling period $10^{-5}$. During the simulations the value of the implicit Lyapunov function has been calculated on-line using bisection method.

It was expectable that relay finite-time stabilizing algorithm should be a sort of (high order) sliding mode control. This fact is indirectly confirmed by simulations, since the chattering phenomenon appear. Fortunately, the oscillations are observed only for the current $i_{L}$. They are not destructive, since $i_{K}$ is the fast variable of the system.

\section{CONCLUSION}

The paper presents relay feedback control algorithm for stabilization of linear multi-input system provided nonasymptotic transitions. The control design procedure combines the ILF method and convex embedding technique. This approach allows us to develop a simple procedure for implicit switching surface design using LMIs. The algorithm of practical implementation of the obtained implicit relay feedback is presented and tested on the numerical example. Its sampled-time analysis is considered as the subject for future research.

\section{APPENDIX}

\section{A. Transformation to Block Form}

Let us denote by rown $(W)$ the number of rows of a matrix $W$ and by $\operatorname{null}(W)$ the matrix that has the columns defining an orthonormal basis of the null space of a matrix $W$.

Let the orthogonal matrices $T_{i}$ be defined by the following simple algorithm:

Initialization : $A_{0}=A, B_{0}=B, T_{0}=I_{n}, k=0$.

Loop: While $\operatorname{rank}\left(B_{k}\right)<\operatorname{rown}\left(A_{k}\right)$ do

$$
\begin{aligned}
& A_{k+1}=B_{k}^{\perp} A_{k}\left(B_{k}^{\perp}\right)^{T}, \quad B_{k+1}=B_{k}^{\perp} A_{k} \hat{B}_{k}, \\
& T_{k+1}=\left(\begin{array}{c}
B_{k}^{\perp} \\
\hat{B}_{k}
\end{array}\right), \quad k=k+1,
\end{aligned}
$$

where $B_{k}^{\perp}=\left(\operatorname{null}\left(B_{k}^{T}\right)\right)^{T}, \hat{B}_{k}=\left(\operatorname{null}\left(B_{k}^{\perp}\right)\right)^{T}$.

It was proven (see, e.g. [35]) that

$$
\begin{aligned}
& G A G^{T}=\left(\begin{array}{ccccc}
A_{11} & A_{12} & 0 & \ldots & 0 \\
A_{21} & A_{22} & A_{23} & \ldots & 0 \\
\ldots & \ldots & \ldots & \ldots & \ldots \\
A_{k-11} & A_{k-12} & \ldots & A_{k-1 k-1} & A_{k-1 k} \\
A_{k 1} & A_{k 2} & \ldots & A_{k k-1} & A_{k k}
\end{array}\right), \\
& \tilde{B}=G B=\left(\begin{array}{ccccc}
0 & 0 & \ldots & 0 & A_{k k+1}^{T}
\end{array}\right)^{T}, \\
& G=\left(\begin{array}{cc}
T_{k} & 0 \\
0 & I_{w_{k}}
\end{array}\right)\left(\begin{array}{cc}
T_{k-1} & 0 \\
0 & I_{w_{k-1}}
\end{array}\right) \ldots\left(\begin{array}{cc}
T_{2} & 0 \\
0 & I_{w_{2}}
\end{array}\right) T_{1} \text {, }
\end{aligned}
$$

where $w_{i}:=n-\operatorname{rown}\left(T_{i}\right), A_{k k+1}=\hat{B}_{0} B_{0}, A_{i j} \in \mathbb{R}^{n_{i} \times n_{j}}$, $n_{i}:=\operatorname{rank}\left(B_{k-i}\right), i, j=1,2, \ldots, k$ and $\operatorname{rank}\left(A_{i+1}\right)=n_{i}$.

Recall that the $B$ has full column $\operatorname{rank}(\operatorname{rank}(B)=$ $m$ ). Consequently, $A_{k k+1}$ is square and nonsingular. Since $\operatorname{rank}\left(A_{i i+1}\right)=n_{i}=\operatorname{rown}\left(A_{i i+1}\right)$ then $A_{i i+1} A_{i i+1}^{T}$ is invertible and $A_{i i+1}^{+}=A_{i i+1}^{T}\left(A_{i i+1} A_{i i+1}^{T}\right)^{-1}$ is the right inverse matrix of $A_{i i+1}$. Introduce the linear coordinate transformation $s=\Phi y, s=\left(s_{1}, \ldots, s_{k}\right)^{T}, s_{i} \in \mathbb{R}^{n_{i}}$, $y=\left(y_{1}, \ldots, y_{k}\right)^{T}, y_{i} \in \mathbb{R}^{n_{i}}$ by the formulas:

$$
\begin{gathered}
s_{i}=y_{i}+\varphi_{i}, \quad i=1,2, \ldots, k, \quad \varphi_{1}=0 \\
\varphi_{i+1}=A_{i i+1}^{+}\left(\sum_{j=1}^{i} A_{i j} y_{j}+\sum_{r=1}^{i} \frac{\partial \varphi_{i}}{\partial y_{r}} \sum_{j=1}^{r+1} A_{r j} y_{j}\right) .
\end{gathered}
$$

The presented coordinate transformation is linear and nonsingular. The inverse transformation $y=\Phi^{-1} s$ is defined as follows: $y_{i}=s_{i}+\psi_{i}, \quad i=1,2, \ldots, k, \quad \psi_{1}=0$, $\psi_{i+1}=A_{i i+1}^{+}\left(\sum_{k=1}^{i} \frac{\partial \psi_{i}}{\partial s_{k}} A_{i i+1} s_{k+1}-\sum_{j=1}^{i} A_{i j}\left(s_{j}+\psi_{j}\right)\right)$.

Applying the transformation $s=\Theta x$ with $\Theta=\Phi G$ to (1) we derive $\dot{s}=\left(\begin{array}{cccc}0 & A_{12} & \ldots & 0 \\ \ldots & \ldots & \ldots & \ldots \\ 0 & \ldots & \ldots & A_{k-1 k} \\ \tilde{A}_{k 1} & \ldots & \ldots & \tilde{A}_{k k}\end{array}\right) s+\tilde{B} u+\Theta d$, that is equivalent to (8) with $K_{l i n}=\tilde{B} A_{k k+1}^{-1}\left(\tilde{A}_{k 1} \ldots \tilde{A}_{k k}\right)$.

\section{B. Proof of Theorem 4}

The proof is divided into several steps. First, based on implicit Lyapunov function method we design a continuous feedback ensuring global finite-time stabilization. Next, we provide an estimation of the domain in which the continuous feedback may be re-configured as a relay using convex embedding procedure. We continue with the Filippov regularization of the closed-loop relay system. At last, we show the local finite-time stability of the closed-loop system when Filippov solutions are being considered. The construction of the switching surface $\Gamma$ is the most specific part of proof, since, usually [20], it is depended on the Lyapunov function, which does not have an explicit representation in our case.

1) The continuous feedback law providing finite-time stabilization: In the proof we use the a result presented in [30]. Let $\mu \in(0,1), \alpha>0, X \in \mathbb{R}^{n \times n}$ and $Y \in \mathbb{R}^{n_{k} \times n}$ satisfy the system of matrix inequalities (13). Let $\Theta \in \mathbb{R}^{n \times n}$ be given by (7), $K:=Y X^{-1}$ and the positive definite function 
$V: \mathbb{R}^{n} \rightarrow \mathbb{R}$ satisfying $Q(V(s), s)=0$ with $Q$ defined by (11) and $P:=X^{-1}$. It is known [30] that the function $V$ is smooth outside the origin and the continuous feedback law

$$
\begin{gathered}
u_{\text {con }}(x(t)) \stackrel{\text { def }}{=} \tilde{u}(\Theta x(t)), \\
\tilde{u}(s)=V^{1-\mu}(s) K D_{r}\left(V^{-1}(s)\right) s,
\end{gathered}
$$

ensures the inequality

$$
\frac{\partial V}{\partial s}\left(\tilde{A} s+\tilde{B} u_{c o n}(s)+\delta\right) \leq-(\alpha-\beta) V^{1-\mu}(s),
$$

for any $\delta$ satisfying (14). Hence, for $V_{\max }=\infty$ the origin of the closed-loop system (1) is globally finite-time stable and the settling-time function is bounded as follows

$$
T\left(x_{0}\right)=\frac{V_{0}^{\mu}}{\mu(\alpha-\beta)},
$$

where $V_{0} \in \mathbb{R}_{+}: Q\left(V_{0}, \Phi G x_{0}\right)=0$. The function $V$ is the Lyapunov function of the closed-loop system [30], which is defined implicitly by means of equation $Q(V, s)=0$. If $V_{\max }<+\infty$ then the given feedback law guarantees local finite-time stabilization for $x_{0} \in \varepsilon\left(V_{\max }\right)$, where $\varepsilon\left(V_{\max }\right)$ is the level set of the Lyapunov function $V$ (see, Corollary 3).

2) Estimation of attraction domain: As follows we show how the continuous feedback (23), (24) can be re-configured as a relay feedback using convex optimization arguments. The representation of the continuous feedback (23), (24) as a convex combination of the relay vectors $v_{i}, i=1, \ldots, N$,

$$
u_{\text {con }}(s)=\sum_{i=1}^{N} \alpha_{i}(s) v_{i}, \quad \alpha_{i}(s) \geq 0, \quad \sum_{i=1}^{N} \alpha_{i}(s)=1
$$

can be applied locally for this purpose. In contrast to the feedback law (23), (24) the relay control (4) is always bounded. Therefore, the representation (27) holds locally in the domain defined as follows

$$
\mathcal{C}_{\mathcal{U}}=\left\{z \in \mathbb{R}^{n}: u_{\text {con }}(z) \in \operatorname{co}(\mathcal{U})\right\} .
$$

Since $u_{c o n}$ is the continuous function [30] and $u_{c o n}(0)=$ 0 , then $\mathcal{C}_{\mathcal{U}}$ is a nonempty compact set. Moreover, due to (3) the inclusion $0 \in \operatorname{int}\left\{\mathcal{C}_{\mathcal{U}}\right\}$ holds. Let $\bar{V} \in \mathbb{R}_{+}$be defined as

$$
\bar{V}=\min \left\{V_{\max }, \sup _{V \in \mathbb{R}_{+}: \varepsilon(V) \subset \mathcal{C}_{\mathcal{U}}} V\right\},
$$

where $\bar{V}>0$ due to $0 \in \operatorname{int}\left\{\mathcal{C}_{\mathcal{U}}\right\}$. Below we show that $\varepsilon(\bar{V})$ is the finite-time attraction domain of the system (1),(4),(15).

3) Filippov regularization of the closed-loop relay system: Since the closed-loop system system (1), (4), (15) has a discontinuous right-hand side, classical solutions may not exists. In order to describe the system behavior, we considered solution $x(t)$ in the sense of Filippov and construct the differential inclusion

$$
\begin{gathered}
\dot{x}(t) \in F(t, x(t)), \\
F(t, x)=\left\{A x+B c o\left(\underset{v \in \mathcal{U}}{\operatorname{argmin}} \Gamma^{T}(t, x) v\right)+\Theta^{-1} \Delta(t, \Theta x)\right\} .
\end{gathered}
$$
Obviously, the obtained inclusion contains all Filippov solutions of the closed-loop system (1), (4), (15). For its finitetime stability analysis Theorem 2 can be utilized.
4) Finite-time stability of the closed-loop relay system: Let us show that the function the implicit Lyapunov function (11) satisfying (25) is also Lyapunov function for the system (1) closed by the relay feedback (4), (15). It is sufficient to show that

$$
\frac{\partial V}{\partial s}(\tilde{A} s+\delta)+\sup _{z \in c o\left(\underset{v \in \mathcal{U}}{\operatorname{argmin}} \tilde{\Gamma}^{T}(s) v\right)} \frac{\partial V}{\partial s} \tilde{B} z \leq-(1-\beta) V^{1-\mu}(s)
$$

for all $\delta \in \Delta(t, s)$ and all $s \in \mathbb{R}^{n} \backslash\{0\}: s \in \varepsilon(\bar{V})$, where

$$
\tilde{\Gamma}(s)=\left(s^{T} D_{r}\left(V^{-1}(s)\right) P D_{r}\left(V^{-1}(s)\right) \tilde{B}\right)^{T} .
$$

Obviously, $\Gamma(t, x)=\tilde{\Gamma}(\Theta x)$ (see, the formula (15)). Recall that the implicit function theorem [33] gives $\frac{\partial V}{\partial s}=$ $-\left[\frac{\partial Q}{\partial V}\right]^{-1} \frac{\partial Q}{\partial x}$. So, we derive the following representation:

$$
\frac{\partial V}{\partial s}=2 V(s) \frac{s^{T} D_{r}\left(V^{-1}(s)\right) P D_{r}\left(V^{-1}(s)\right)}{s^{T} D_{r}\left(V^{-1}(s)\right)\left(H_{\mu} P+P H_{\mu}\right) D_{r}\left(V^{-1}(s)\right) s},
$$

where $H_{\mu} P+P H_{\mu}>0$ (see, (13)). Hence, we conclude that the relation

$$
\underset{v \in \mathcal{U}}{\operatorname{argmin}} \tilde{\Gamma}^{T}(s) v=\underset{v \in \mathcal{U}}{\operatorname{argmin}} \frac{\partial V}{\partial s} \tilde{B} v
$$

holds for any $s \in \mathbb{R}^{n} \backslash\{0\}$. Moreover, the inequality $\frac{\partial V}{\partial s} \tilde{B} z \leq \frac{\partial V}{\partial s} \tilde{B} v_{i}$, holds for any $v_{i} \in \mathcal{U}$ and any $z \in$ $\underset{\partial s}{\operatorname{argmin}} \tilde{\Gamma}^{T}(s) v$. Using the standard convexity arguments, it $v \in \mathcal{U}$

can be easily shown that this inequality also holds for any $z \in c o\left(\underset{v \in \mathcal{U}}{\operatorname{argmin}} \tilde{\Gamma}^{T}(s) v\right)$. Therefore, we derive

$$
\frac{\partial V}{\partial s} \tilde{B} z=\sum_{i=1}^{N} \tilde{\alpha}_{i} \frac{\partial V}{\partial s} \tilde{B} z \leq \sum_{i=1}^{N} \tilde{\alpha}_{i} \frac{\partial V}{\partial s} \tilde{B} v_{i}
$$

$\forall \tilde{\alpha}_{i} \geq 0: \sum_{i=1}^{N} \tilde{\alpha}_{i}=1$ and $\forall z \in c o\left(\underset{v \in \mathcal{U}}{\operatorname{argmin}} \tilde{\Gamma}^{T}(s) v\right)$.

Finally, taking into account the representation (27) of the control law (23), (24) and the equality (25) we derive with $\tilde{\alpha}_{i}=\alpha_{i}(s), i=1, \ldots, N$, the representation

$$
\frac{\partial V}{\partial s}(\tilde{A} s+\delta)+\sum_{i=1}^{N} \alpha_{i}(s) \frac{\partial V}{\partial s} \tilde{B} v_{i} \leq-(\alpha-\beta) V^{1-\mu},
$$

$\sum_{i=1}^{N} \alpha_{i}(s)=1, \alpha_{i}(s) \geq 0$ for any $\delta \in \Delta(t, s)$ and any $s \in \varepsilon(\bar{V})$. The obtained representation completes the proof.

\section{Proof of Corollary 8}

In [30] it was proven that for any fixed $V_{i} \in \mathbb{R}_{+}$the control (23), (24) with $V=V_{i}$ is the linear stabilizing feedback for the system (8) and the corresponding quadratic Lyapunov function $V_{i}$ is defined as follows $V_{i}(s)=s^{T} P_{i} s$, where $P_{i}:=$ $D_{r}\left(V_{i}^{-1}\right) P D_{r}\left(V_{i}^{-1}\right)>0$. Hence, the ellipsoid $\varepsilon\left(V_{i}\right)$ defined in Corollary 3 is strictly positively invariant set.

Corollary 9 ([30]): If 1) the conditions (13) of Theorem 4 hold; 2) $\left\{t_{i}\right\}_{i=0}^{+\infty}$ is an arbitrary sequence of time instances such that $0=t_{0}<t_{1}<t_{2}<\ldots$ and $\lim _{i \rightarrow+\infty} t_{i}=$ $+\infty$; 3) the control has the form (23) with $\tilde{u}(s)=\tilde{u}_{V_{i}}(s)$ on each time interval $\left[t_{i}, t_{i+1}\right)$, where $\tilde{u}_{V}(s)$ is defined by (24) and $V_{i} \in R_{+}: Q\left(V_{i}, s\left(t_{i}\right)\right)=0$. Then the closed-loop system (1) is globally asymptotically stable. 
The convex embedding technique for design relay feedback law using existing linear stabilizing feedback is studied in [20]. Applying this technique to the switching linear feedback described in this corollary (see the condition 3)) we finish the proof of Corollary 8. Note that the ellipsoids $\varepsilon\left(V_{i}\right)$ are also strictly positively invariant for the relay feedback.

\section{Proof of Proposition 6}

For proof of this proposition we use the ideas presented in [30]. Obviously, $\gamma I_{n} \leq P^{1 / 2} H_{\mu} P^{-1 / 2}+P^{-1 / 2} H_{\mu} P^{1 / 2}$ or equivalently $\gamma P \leq P H_{\mu}+H_{\mu} P$. On the one hand, we have $\gamma=\gamma s^{T} D_{r}\left(V^{-1}\right) P D_{r}\left(V^{-1}\right) s \leq s^{T} D_{r}\left(V^{-1}\right)\left(P H_{\mu}+\right.$ $\left.H_{\mu} P\right) D_{r}\left(V^{-1}\right) s$, where $(V, s) \in \mathbb{R}_{+} \times \mathbb{R}^{n}$ such that $Q(V, s)=0$. On the other hand,

$$
\begin{gathered}
1=s^{T} D_{r}\left(V^{-1}\right) P D_{r}\left(V^{-1}\right) s \geq \\
\left\{\begin{array}{cll}
\lambda_{\min }(P) V^{-2} s^{T} s & \text { for } \quad & s^{T} P s \leq 1, \\
\lambda_{\min }(P) V^{-2-2(k-1) \mu} s^{T} s & \text { for } \quad & s^{T} P s>1 .
\end{array}\right.
\end{gathered}
$$

Hence, we derive $\delta^{T} D_{r}\left(V^{-1}\right) R^{-1} D_{r}\left(V^{-1}\right) \delta=$ $\sum_{i=1}^{k} \beta_{i}^{-1} V^{-2-2(k-i) \mu} \delta^{T} E_{i} \delta \leq \frac{\gamma}{V^{2 \mu}}\left(\beta_{1}+\ldots+\beta_{k}\right) \leq$ $\beta V^{-2 \mu} s^{T} D_{r}\left(V^{-1}\right)\left(P H_{\mu}+H_{\mu} P\right) D_{r}\left(V^{-1}\right) s$, i.e. the inequality (14) holds.

\section{E. Proof of Proposition 7}

If $\mu=1$ then the control function $\tilde{u}_{v}$ is continuous outside the origin and bounded for all $x \in \mathbb{R}^{n}$. Indeed, since $s^{T} D_{r}\left(V^{-1}\right) P D_{r}\left(V^{-1}\right) s=1 \rightarrow\left\|D_{r}\left(V^{-1}\right) s\right\|^{2} \leq \frac{1}{\lambda_{\min }(P)}$ and $\tilde{u}^{2}(s) \leq s D_{r}\left(V^{-1}\right) K^{T} K D_{r}\left(V^{-1}\right) s \leq \frac{\lambda_{\max }\left(K^{T} K\right)}{\lambda_{\min }(P)}$.

Hence, in order to restrict globally the control magnitude by $\|\tilde{u}(s)\| \leq u_{0}$ the inequality $K^{T} K \leq u_{0}^{2} P$ should be added to (13). Therefore, the inequality (19) guarantees that $\mathcal{C}_{\mathcal{U}}$ is nonempty and all steps of the proof of Theorem 4 can be repeated for $\mu=1$. Moreover, $\left\{s \in \mathbb{R}^{n}:\left\|u_{\text {con }}(s)\right\| \leq\right.$ $\left.u_{\max }\right\} \subset \mathcal{C}_{\mathcal{U}}$, so $\bar{V}=\min \left\{V_{\max },+\infty\right\}=V_{\max }$.

\section{REFERENCES}

[1] I. Flugge-Lotz, Discontinuous automatic control. Princeton University Press, 1953

[2] Y. Z. Tsypkin, Relay Control Systems. Moscow: Nauka, 1984.

[3] I. Boiko, Discontinuous Control Systems: Frequency-Domain Analysis and Design. Springer, 2009.

[4] V. Utkin, J. Guldner, and J. Shi, Sliding Mode Control in ElectroMechanical Systems. CRC Press., 2009.

[5] Y. Orlov, "Finite time stability and robust control synthesis of uncertain switched systems," SIAM Journal of Control and Optimization, vol. 43(4), pp. 1253-1271, 2005.

[6] A. Levant, "Principles of 2-sliding mode design," Automatica, vol. 43, pp. 576-586, 2007.

[7] G. Bartolini, A. Pisano, E. Punta, and E. Usai, "A survey of applications of second-order sliding mode control to mechanical systems," International Journal of Control, vol. 76(9/10), pp. 875-892, 2003.

[8] A. Polyakov and A. Poznyak, "Unified Lyapunov function for a finitetime stability analysis of relay second-order sliding mode control systems," IMA Journal of Mathematical Control and Information, vol. 29(4), pp. 529-550, 2012.

[9] B. Drazenovic, "The invariance conditions in variable structure systems," Automatica, vol. 5, no. 3, pp. 287-295, 1969.

[10] G. S. Deaecto, J. C. Geromel, F. S. Garcia, and J. A. Pomilio, "Switched affine systems control design with application to dc-dc converters," IET Control Theory \& Applications, vol. 4, no. 4, pp. 1201-1210, 2010.
[11] S. L. Brunton and B. R. Noack, "Closed-loop turbulence control: Progress and challenges," Applied Mechanics Reviews, vol. 67, no. 5, p. 050801, 2015.

[12] M. Feingesicht, C. Raibaudo, A. Polyakov, F. Kerhervé, and J.-P. Richard, "A bilinear input-output model with state-dependent delay for separated flow control," in European Control Conference 2016, Aalborg, 2016.

[13] A. Van Der Schaft and H. Schumacher, An introduction to hybrid dynamical systems. Springer, 2000.

[14] D. Liberzon, Switching in Systems and Control. Birkhauser, 2003.

[15] G. Chesi, P. Colaneri, J. C. Geromel, R. Middleton, and R. Shorten, "A nonconservative lmi condition for stability of switched systems with guaranteed dwell time," IEEE Transactions on Automatic Control, vol. 57, no. 5, pp. 1297-1302, 2012.

[16] R. Shorten, F. Wirth, O. Mason, K. Wulff, and C. King, "Stability criteria for switched and hybrid systems," Invited paper for SIAM Review, vol. 49, no. 4, pp. 545-592, 2007.

[17] P. Bolzern and W. Spinelli, "Quadratic stabilization of a switched affine system about a nonequilibrium point," in Proceeding of the 2004 American Control Conference, Boston, Massachusetts, USA, 2004.

[18] G. Deaecto, J. Geromel, F. Garcia, and J. Pomilio, "Switched affine systems control design with application to dc-dc converters," Control Theory Applications, IET, vol. 4, no. 7, pp. $1201-1210$, july 2010.

[19] X. Xu and G. Zhai, "Practical stability and stabilization of hybrid and switched systems," IEEE Transactions on Automatic Control, vol. 50, no. 11, pp. 1897-1903, 2005.

[20] L. Hetel, E. Fridman, and T. Floquet, "Variable structure control with generalized relays: A simple convex optimization approach," IEEE Transactions on Automatic Control, vol. 60(2), pp. 497-502, 2015.

[21] S. Bacha, I. Munteanu, and A. Bratcu, Power Electronic Converters Modeling and Control: with Case Studies. Springer, 2014.

[22] V. Korobov, "A solution of the problem of synthesis using a controllability function," Doklady Academii Nauk SSSR, vol. 248, pp. 10511063, 1979.

[23] — " "A general approach to the solution of the bounded control synthesis problem in a controllability problem," Math. USSR Sbornik, vol. 37, no. 4, pp. 535-557, 1979.

[24] J. Adamy and A. Flemming, "Soft variable-structure controls: a survey," Automatica, vol. 40, pp. 1821-1844, 2004.

[25] A. Polyakov, D. Efimov, and W. Perruquetti, "Finite-time and fixedtime stabilization: Implicit lyapunov function approach," Automatica, vol. 51, pp. 332-340, 2015.

[26] A. Polyakov and L. Hetel, "On finite-time stabilization via relay control," in Conference on Decision and Control, 2015, pp. 40984102.

[27] G. Bartolini, E. Punta, and T. Zolezzi, "Simplex sliding mode control of multi-input systems with chattering reduction and mono-directional actuators," Automatica, vol. 47, pp. 2433-2437, 2011.

[28] S. V. Bajda and D. B. Izosimov, "Vector method of design of sliding motion and simplex algorithms," Automation and Remote Control, vol. 46, pp. 830-837, 1985.

[29] A. Filippov, Differential equations with discontinuous right-hand sides. Kluwer, Dordrecht, 1988.

[30] A. Polyakov, D. Efimov, and W. Perruquetti, "Robust stabilization of mimo systems in finite/fixed time," International Journal of Robust and Nonlinear Control, vol. 26, no. 1, pp. 69-90, 2016.

[31] E. Roxin, "On finite stability in control systems," Rendiconti del Circolo Matematico di Palermo, vol. 15(3), pp. 273-283, 1966.

[32] S. Bhat and D. Bernstein, "Finite-time stability of continuous autonomous systems," SIAM Journal of Control and Optimization, vol. 38(3), pp. 751-766, 2000.

[33] R. Courant and F. John, Introduction to calculus and analysis (Vol. II/1). New York: Springer, 2000.

[34] S. Drakunov, D. Izosimov, A. Lukyanov, V. Utkin, and V. Utkin, "Block control principle I," Automation and Remote Control, vol. 51(5), pp. 601-609, 1990.

[35] A. Polyakov, "Nonlinear feedback design for fixed-time stabilization of linear control systems," IEEE Transactions on Automatic Control, vol. 57(8), pp. 2106-2110, 2012.

[36] A. Polyakov and I. Chairez, "A new homogeneous quasi-continuous second order sliding mode control," in Latinamerican Congress on Automatic Control, Cancun, Mexico, 2014. 